\title{
5-Merkapto-2-(3-metil-tiyofen-2-il-metilidenamino)-1,3,4-tiyadiazol Bileşiğinin Sentezi, Karakterizasyonu ve DFT Hesaplamaları
}

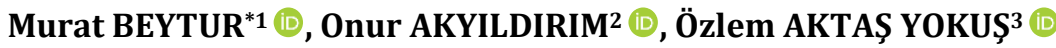 \\ ${ }^{1}$ Kafkas Üniversitesi, Fen-Edebiyat Fakültesi, Kimya Bölümü, 36100, Kars, Türkiye \\ ${ }^{2}$ Kafkas Üniversitesi, Mühendislik Mimarlık Fakültesi, Kimya Mühendisliği Bölümü, 36100, Kars, Türkiye \\ ${ }^{3}$ Kafkas Üniversitesi, Eğitim Fakültesi, Matematik ve Fen Bilimleri Eğitimi Bölümü, 36100, Kars, Türkiye
}

(Alınış / Received: 21.12.2018, Kabul / Accepted: 12.07.2019, Online Yayınlanma / Published Online: 30.08.2019)

\section{Anahtar Kelimeler B3LYP, \\ GIAO, \\ Tiyadiazol, \\ Polarizebilite, \\ Hiperpolarizebilite, 6-311G+(d,p)}

\begin{abstract}
Özet: 5-Merkapto-2-(3-metil-tiyofen-2-il-metiliden-amino)-1,3,4-tiyadiazol bileşiği sentezlenmiş ve IR, ${ }^{1} \mathrm{H}-\mathrm{NMR}$ ve ${ }^{13} \mathrm{C}$-NMR spektroskopik yöntemleri ile karakterize edilmiștir. Sentezlenen bileșiğin geometrik optimizasyonu Gaussian G09W yazılımı kullanılarak DFT/B3LYP yöntemi ve 6-311G+(d,p) temel seti yapılmıştır. Elde edilen optimize yapıdan molekülün geometrik yapıları, titreşim dalga sayıları, ${ }^{1} \mathrm{H}$ ve ${ }^{13} \mathrm{C}$ nükleer manyetik rezonans kimyasal kayma değerlerinin kuantum kimyasal hesaplamaları elde edilmiştir. Bu değerler ile deneysel spektroskopik değerler karşılaştırılmıştır. Teorik değerlerin deneysel değerleri desteklediği gözlemlenmiştir. İlaveten, ilgili bileşiğin, çizgisel olmayan optik özellikleri; tek nokta enerji hesabında polar hesapları yapılarak polarizebilite ve hiperpolarizebilite değerleri hesaplanmıştır. Son olarak, Mulliken atomik yükleri, HOMO-LUMO enerjileri, dipol momentleri, toplam enerjileri, iyonlașma potansiyeli, elektron ilgisi, moleküler yumuşaklık, moleküler sertlik ve elektronegatifliği aynı metotlar ve aynı set kullanılarak hesaplanmıștır.
\end{abstract}

\section{Synthesis, Characterization and DFT Calculations of 5-Mercapto-2-(3-methyl- thiophene-2-yl-methylidenamino)-1,3,4-thiadiazole}

Keywords

B3LYP,

GIAO,

Thiadiazole,

Polarizability,

Hyperpolarizability

$6-311 G+(d, p)$

\begin{abstract}
Mercapto-2-(3-methyl-thiophene-2-yl-methylidenamino)-1,3,4-thiadiazole compound was synthesized, and it was characterized by IR, ${ }^{1} \mathrm{H}-\mathrm{NMR}$ and ${ }^{13} \mathrm{C}-\mathrm{NMR}$ spectroscopic methods. Geometrical optimization of the synthesized compound was carried out by DFT/B3LYP method and $6-311 \mathrm{G}+(\mathrm{d}, \mathrm{p})$ basic sets by using Gaussian GO9W computer software. From the optimized structure, the geometric structures of the molecule, vibration wave numbers, quantum chemical calculations of ${ }^{1} \mathrm{H}$ and ${ }^{13} \mathrm{C}$ nuclear magnetic resonance chemical shift values were obtained. These values were compared with experimental spectroscopic values. It was observed that theoretical values support the experimental values. Additionally, polarizability and hyperpolarizability values of non-linear optical characteristic of the related compounds were calculated using polar calculations in single point energy account. Finally, Mulliken atomic charges, HOMO-LUMO energies, dipol moments, total energies, ionization potential, electron information, molecular softness, molecular stiffness and electronegative characteristics were calculated by using the same methods and same sets.
\end{abstract}

\section{Giriş}

Beş üyeli heterosiklik halka içeren bileşiklerin olağanüstü kimyasal davranıș sergilediği ve çok yönlü biyolojik aktivitelerin geniş bir spektrum sergilediği gözlenmiştir. Tiyadiazol kükürt ve azot atomu içeren beş üyeli bir halka sistemidir. Tiyadiazol, 1,2,3tiyadiazol, 1,2,5-tiyadiazol, 1,2,4-tiyadiazol ve 1,3,4tiyadiazol gibi izomerleri olan bir heterosiklik bileşiktir. 1,3,4-Tiyadiazol en çok çalışılan izomerdir. Farklı özelliklere sahip beş üyeli heterosiklik bileşiklerin sentezlenmesi için birçok farklı türeve dönüștürülmüștür [1,2]. Farklı özelliklere sahip beș üyeli heterosiklik bileşiklerin sentezlenmesi için literatürde birçok farklı yöntem yayınlanmıştır [1, 2]. Eşsiz bir yapı olan 1,3,4-tiyadiazol, heterosiklik kimya ve tıbbi kimyada önemli bir motiftir [3]. Örneğin, 1,3,4-tiyadiazol kısımları içeren birçok ilaç piyasada 
mevcuttur, örneğin, asetazolamid, methazolamid, sülfamethazol [4], Desaglybuzole (antidiyabetik), Furidiazin (antimikrobiyal) ve Butazolamid (diüretik) ticari olarak temin edilebilen yapısında 1,3,4tiyadiazol grubu bulunduran ilaçlardır. 1,3,4Tiyadiazol türevleri antimikrobiyal [5], antikanser [6, 7], antipsikotik [8], antitüberküler [9], antihistamin [10], antikonvülsan [11], antiinflamatuar [12], antilishmanyal [13], anti-hepatit B viral [14], antiparkinson [15] ve antidiyabetik [16] gibi birçok uygulama alanlarına sahiptir. Tiyadiazol halkasında N=C-S yapısının varlığl, belirtilen çoklu aktiviteler içermektedir. Tiyadiazol kısmı "hidrojen bağlama bölgesi" ve "iki elektron verici sistem" olarak görev yapar. Ayrıca, tiyadiazollerin aromatikliği düşük toksisiteye ve in-vivo dayanıklılığa katkıda bulunur [6]. 1,3,4-Tiyadiazolün kükürt atomu, gelişmiş liposolubilite sağlar ve 1,3,4-tiyadiazolün mezoiyonik doğası, bu bileşik sınıfının, iyi bir doku geçirgenliğine sahip olmasını sağlar. 1,3,4-tiyadiazol-2-tiyollerin farmakofor aktivitesi yaygin olarak bilinmektedir [17].

Schiff bazları, bir primer aromatik amin ve farklı aldehit veya keton arasinda bir kondenzasyon reaksiyonu gerçekleştirilerek hazırlanmıştır. Bunlar ilaç ve farmasötik alanda hayati bir rol oynayan azometin grubuna (NCH) sahip önemli bir biyoaktif molekül sınıfıdır. Bu nedenle, Schiff bazlarının tüm türevleri terapötik olarak aktiftir ve birçok yayında rapor edilmiştir. Çok çeşitli endüstriyel ve farmakolojik uygulamalara sahiptirler. Farklı 2amino substitüe 1,3,4-tiyadiazoller ve bunların Schiff bazı türevleri biyolojik ve farmakolojik aktiviteye sahip olduğu belirlenmiştir [18]. Schiff bazı bağlı 1,3,4-Tiyadiazol türevleri, antikanser, antibakteriyel, antidepresan, antidiyabetik ve antifungal aktiviteleri bile sergiler [19].

\section{Materyal ve Metot}

\subsection{Deneysel}

$\mathrm{Bu}$ çalışmada, kimyasal reaktifler ve bütün çözücüler Merck AG, Aldrich ve Fluka firma distribütörlerinden satın alınmıştır. Sentezlenen bileşiğin erime noktası Stuart melting point SMP30 marka erime noktası cihazı kullanılarak belirlenmiştir. IR spektrumu ALPHA-P BRUKER FT-IR spektrometresinde alınmıştır. ${ }^{1} \mathrm{H}-\mathrm{NMR}$ ve ${ }^{13} \mathrm{C}-\mathrm{NMR}$ spektrumları BRUKER ULTRASHIELD PLUS BIOSPIN marka 400 MHz'lik NMR cihazında alınmıștır.

5-Merkapto-2-(3-metil-tiyofen-2-il-metilidenamino)-1,3,4-tiyadiazol (1): 2-Amino-5-merkapto1,3,4-tiyadiazol'ün $(10 \mathrm{mmol})$ glacial asetik asit çözeltisi $(20 \quad \mathrm{ml}, \quad \% 100)$ 3-metil-2-tiyofenkarboksialdehid (10 mmol) ile muamele edildi ve 2 saat reflux edilip süzüldü. Süzüntü etanolden $3 \mathrm{kez}$

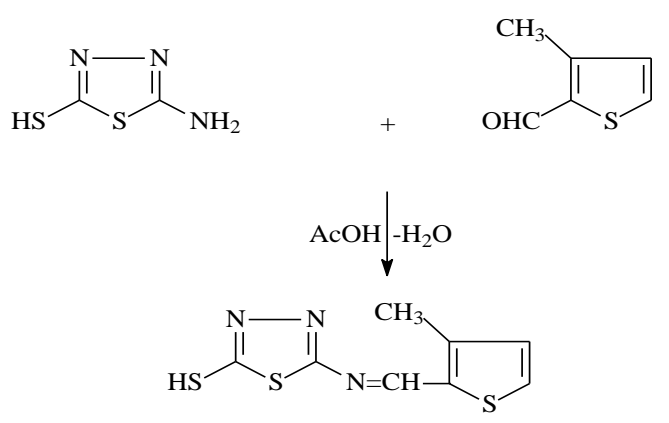

Şekil 1. Bileşik 1'in sentez yöntemi

kristallendirildi. Verim: $1.67 \mathrm{~g} \mathrm{(70 \% );} \mathrm{mp:} 223{ }^{\circ} \mathrm{C}$; IR $\left(\mathrm{KBr}, \mathrm{v}, \mathrm{cm}^{-1}\right): 2585(\mathrm{SH}), 1581(\mathrm{C}=\mathrm{N}), 2670(\mathrm{SH}) ;{ }^{1} \mathrm{H}$ NMR (400 MHz, DMSO-d $): \delta 2.50\left(\mathrm{~s}, 3 \mathrm{H}, \mathrm{CH}_{3}\right), 7.12$ (d, 1H, ArH; J=4.80 Hz), 7.96 (d, 1H, ArH; J=5.20 Hz), 8.74 (s, 1H, N=CH), 14.41 (s, 1H, SH); ${ }^{13} \mathrm{C}$ NMR (100 $\mathrm{MHz}, \quad$ DMSO-d 6$): \delta 14.16\left(\mathrm{CH}_{3}\right), 132.21,134.32$, 134.79, 148.45 (Ar-C), $159.69 \quad(\mathrm{~N}=\mathrm{CH}), \quad 164.39$ (Tiyazol C2), 186.56 (Tiyazol $\mathrm{C}_{5}$ ).

\subsection{Hesaplama detayları}

5-Merkapto-2-(3-metil-tiyofen-2-il-metilidenamino)1,3,4-tiyadiazol'ün moleküler modellemesi Gaussian 09W programında [20] DFT yöntemi ile gerçekleştirilmiştir (Şekil 2). Çılktı dosyaları GaussView 5 yazılımı ile görselleştirilmiştir [21]. DFT (Yoğunluk fonksiyonel teorisi) yöntemi [22, 23] ile 6$311 \mathrm{G}+(\mathrm{d}, \mathrm{p})$ temel seti kullanılarak ilgili molekülün optimize edilmiş geometrisi, elektronik, spektroskopik, termodinamik özellikleri ve lineer olmayan optik özellikleri incelenmiștir. VEDA 4F programı ile detaylı bir titreşim analizi gerçekleştirilmiştir $[24,25] .{ }^{1} \mathrm{H}$ ve ${ }^{13} \mathrm{C} \quad \mathrm{NMR}$ spektrumları, B3LYP ve HF yöntemleriyle 6-311G+(d, p) seviyesinde GIAO'ya (gauge including atomik orbital) göre hesaplanmıştır [26-29]. Sentezlenen yeni bileşiğin birinci dereceden hiperpolarizebilite ve ilişkili özellikleri [30-32], moleküler elektrostatik potansiyel yüzeyi aynı yöntem ve aynı temel set ile elde edilmiştir.

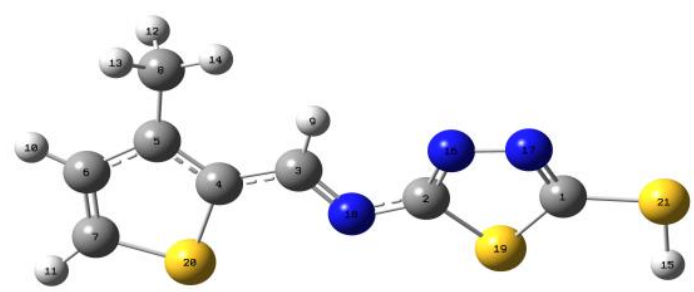

Şekil 2. Sentezlenmiş yeni bileşiğinin (6-311+G(d,p)) gausview görünümü

\section{Bulgular}

\subsection{Elektronik özellikler}

HOMO (en yüksek dolu moleküler orbital) ve LUMO (en düşük dolu olmayan moleküler orbital), kuantum kimyasal hesaplamalar vasitasıyla elektronik çalışmalarda çok önemli parametrelerdir. HOMO 
enerjisi elektron verme yeteneğini, LUMO enerjisi elektron çekme yeteneğini karakterize eder. HOMO ile LUMO arasındaki boşluk, molekülün kimyasal stabilitesini karakterize eder [33]. Sentezlenen yeni bileşiğin toplam enerjisi, HOMO ve LUMO enerjileri (Şekil 3), enerji boşluğu ( $\mathrm{E}=\left(\mathrm{E}_{\text {Lumo }}-\mathrm{E}_{\text {номо }}\right)$ ), iyonizasyon potansiyeli ( $\mathrm{I}=-\mathrm{E}_{\text {номо }}$ ), elektron afinitesi $\left(A=-E_{\text {Lumo }}\right)$, mutlak elektronegatiflik $(\chi=(I+A) / 2)$, mutlak sertlik $(\eta=(I-A) / 2)$ ve yumuşaklık $(\sigma=1 / 2 \eta)$ DFT/B3LYP yöntemine göre $6-311 \mathrm{G}+(\mathrm{d}, \mathrm{p})$ temel seti ile hesaplanmış ve elde edilen sonuç Tablo 1'te verilmiştir.

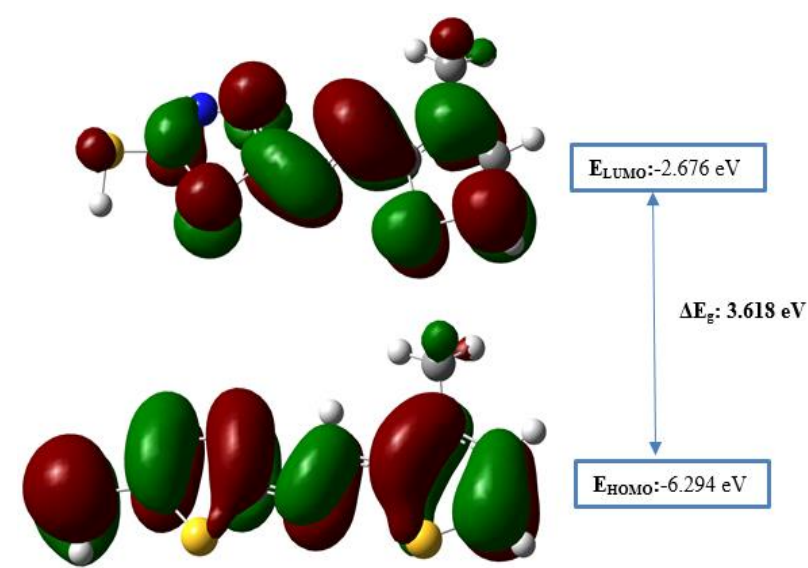

Şekil 3. Molekülün için sınır moleküler yörünge resimleri ve enerjileri

Sentezlenen yeni bileşiğin HOMO ve LUMO enerjileri 6.294ve -2.676 eV olarak hesaplanmış ve HOMO ile LUMO orbital arasındaki enerji boşluğu B3LYP/6311G+(d,p) temel setinde $3.618 \mathrm{eV}$ olarak gözlemlenmiştir. Elde edilen nispeten küçük HOMOLUMO enerji aralığı yük transferinin molekül içinde gerçekleştiğini göstermektedir. İncelenen moleküllerde HOMO-LUMO enerji boşluğu için daha düşük değerin daha polarize, daha reaktif ve daha az kararlı olmasını sağladığı iyi bilinmektedir. Küçük HOMO-LUMO enerji boşluklarına sahip moleküler sistemler de yumuşak molekül olarak adlandırılır. Sınır moleküler orbital boşluğu, bir molekülün elektronegatifliğini, kimyasal sertlik ve yumuşaklığını, kimyasal reaktivitesini ve optik polarizasyonunu karakterize etmede yardımcı olur [34]. Elektronegatiflik, kimyasal sertlik ve kimyasal yumuşaklık HOMO ve LUMO enerji değerleri kullanılarak hesaplanabilir [35]. $\eta, S$ ve $\chi$ parametreleri B3LYP/6-311+(d,p) seviyesi kullanılarak 1.809, 0.904 ve $4.485 \mathrm{eV}$ olarak hesaplanmiștır.

Tablo 1. B3LYP/6-311G+(d,p) seviyesinde elde edilen sentezlenen bileșik için hesaplanmıș elektronik özellikler

\begin{tabular}{|c|c|}
\hline elektronik Özellik & B3LYP (eV) \\
\hline I; İyonlaşma Potansiyeli & 6.294 \\
A; Elektron İlgisi & 2.676 \\
$\boldsymbol{\eta} ;$ Moleküler Sertlik & 1.809 \\
S; Moleküler Yumuşaklık & 0.904 \\
$\boldsymbol{\chi} ;$ elektronegatiflik & 4.485 \\
$\Delta \mathrm{E}$ & 3.618 \\
\hline
\end{tabular}

\subsection{Spektroskopik özellikler}

\subsubsection{Infrared spektrum analizi}

Titreşim analizinin temel amacı, incelenen sistemin özel moleküler yapıları ile bağlantılı titreșim modlarını belirlemektir. Titreşimsel dalga boyları, 6$311+(d, p)$ temel seti ile B3LYP seviyesi kullanılarak hesaplanmıștır. Yapılan analiz sonucunda hesaplanan frekanslar içerisinde negatif frekans gözlemlenmemiştir. Bu sonuç elde ettiğimiz yapının kararlı bir yapı olduğunu göstermektedir. Teorik olarak elde edilen dalgasayıları Tablo 2'de gösterildiği gibi Veda4f programından yararlanılmıştır [36]. Dispersiyon kuvvetlerinin etkisinin önemli olduğu durumlar haricinde, DFT yöntemi çok sayıda bileşiğe uygulanabilir. DFT seviyeleri, deneysel spektruma göre yüksek doğrulukta titreşim spektrumuna sahiptir. Ancak, DFT hesaplarının vakumda serbest bir molekül için gerçekleştirildiği, farklı moleküler içi ve moleküller arası etkileşimlere maruz kalan örnekler yetersiz kaldığı göz önünde bulundurulmalıdır. Ek olarak, DFT düzeylerinin, anhormonikliğin ihmali, elektron korelasyon etkilerinin eksik eklenmesi ve temel set eksiklikleri nedeniyle deneysel olanlara göre daha yüksek dalga boyları verdiği iyi bilinmektedir. $\mathrm{Bu}$ yüzden, yukarıda verilen sistematik hataları düzeltmek için 0,958 ampirik bir ölçekleme faktörü kullanılmıştır [37, 38].

Aromatik sistemlerin, aromatik $\mathrm{CH}$ gerilme titreşimleri nedeniyle $3200-3000 \mathrm{~cm}^{-1}$ bölgesinde genellikle titreşim bantları sergiledikleri iyi bilinmektedir. Sentezlenmiş yeni molekül için kaydedilen FT-IR spektrumunda, 3093 ve 3072 cm1'de gözlemlenen bant aromatik $\mathrm{CH}$ gerilme titreşimidir. Hesaplanan spektrumdaki karşılık gelen bantlar 3108-3069 $\mathrm{cm}^{-1}$ aralı̆̆ında bulunmuștur. Tablo 2'teki Veda4f katkılarından da anlaşıldığı gibi, bu modlar neredeyse \% 100'e katkıda bulunan saf germe titreşimleridir.

\subsection{2. ${ }^{1} \mathrm{H}$ ve ${ }^{13} \mathrm{C}$ NMR spektral analiz}

Aromatik karbon atomlarının 100-150 ppm aralığında NMR sinyali verdiği iyi bilinmektedir [39, 40]. Ancak, elektronegatif atomlara bir koordinasyon halinde, aromatik karbon atomlarından kaynaklanan bu NMR sinyalleri daha yüksek değerlere kaymaktadır. Çalıșmamızda, tiyazol halkasındaki karbon atomlarl, 148.45-132.21 ppm aralıklarında NMR sinyali verir ve bu sinyaller teorik olarak, 158.67-136.22 ppm aralıklarında gözlemlenmiştir. Tiyadiazol halkasındaki karbon atomları C1 ve C2 deneysel olarak 167.39, 186.54 teorik olarak 172.25, 184.49 olarak gözlemlenmiştir. Tiyadiazol halkasındaki karbonların bu kadar düşük alanda çıkmasının sebebi elektronegatif $\mathrm{S}$ ve $\mathrm{N}$ atomlarının yapıda bulunmasından dolayıdır. Metil grubu karbon atomları (C8) deneysel 14.16 teorik ise 14.83 ppm'de sinyaller verir. Aromatik karbon verileri literatürde verilen değerlerle uyumlu olduğu tespit edilmiștir. 
Tablo 2. Molekül için gözlenen ve hesaplanan titreşimsel dalga sayılarının karșılaștırılması ve değerlendirilmesi

\begin{tabular}{|c|c|c|}
\hline Titreşim Türlerinin Değerlendirilmesi & Den*. & DFT* \\
\hline$\tau \mathrm{S}_{20} \mathrm{C}_{7} \mathrm{C}_{6} \mathrm{C}_{5}(38), \tau \mathrm{C}_{3} \mathrm{C}_{5} \mathrm{~S}_{20} \mathrm{C}_{4}(16)$ & 471 & 476 \\
\hline$\tau \mathrm{C}_{1} \mathrm{~N}_{17} \mathrm{~N}_{16} \mathrm{C}_{2}, \tau \mathrm{N}_{16} \mathrm{C}_{2} \mathrm{~N}_{18} \mathrm{C}_{3}, \tau \mathrm{N}_{18} \mathrm{C}_{3} \mathrm{C}_{4} \mathrm{C}_{5}(48), \tau \mathrm{C}_{7} \mathrm{C}_{6} \mathrm{C}_{5} \mathrm{C}_{4}(12)$ & 556 & 588 \\
\hline$v \mathrm{~S}_{19} \mathrm{C}_{1}, v \mathrm{~S}_{21} \mathrm{C}_{1}(48), \delta \mathrm{S}_{19} \mathrm{C}_{1} \mathrm{~N}_{17}, \delta \mathrm{S}_{21} \mathrm{C}_{1} \mathrm{~N}_{17}(11), \tau \mathrm{C}_{1} \mathrm{~N}_{17} \mathrm{~N}_{16} \mathrm{C}_{2}, \tau \mathrm{N}_{16} \mathrm{C}_{2} \mathrm{~N}_{18} \mathrm{C}_{3}, \tau \mathrm{N}_{18} \mathrm{C}_{3} \mathrm{C}_{4} \mathrm{C}_{5}(12)$ & 583 & 599 \\
\hline$v \mathrm{~S}_{20} \mathrm{C}_{7}(24), \delta \mathrm{S}_{20} \mathrm{C}_{7} \mathrm{C}_{6}(20)$ & 633 & 676 \\
\hline$\tau \mathrm{H}_{9} \mathrm{C}_{3} \mathrm{~N}_{18} \mathrm{C}_{2}, \tau \mathrm{H}_{10} \mathrm{C}_{6} \mathrm{C}_{7} \mathrm{~S}_{20}, \tau \mathrm{H}_{11} \mathrm{C}_{7} \mathrm{~S}_{20} \mathrm{C}_{4}(83)$ & 679 & 712 \\
\hline$v \mathrm{~N}_{18} \mathrm{C}_{2}, v \mathrm{C}_{3} \mathrm{C}_{4}, v \mathrm{C}_{6} \mathrm{C}_{5}(10), \delta \mathrm{C}_{2} \mathrm{~N}_{18} \mathrm{C}_{3}(12)$ & 739 & 777 \\
\hline$\tau \mathrm{H}_{9} \mathrm{C}_{3} \mathrm{~N}_{18} \mathrm{C}_{2}, \tau \mathrm{H}_{10} \mathrm{C}_{6} \mathrm{C}_{7} \mathrm{~S}_{20}, \tau \mathrm{H}_{11} \mathrm{C}_{7} \mathrm{~S}_{20} \mathrm{C} 4(81), \tau \mathrm{S}_{20} \mathrm{C}_{7} \mathrm{C}_{6} \mathrm{C}_{5}(11)$ & 832 & 864 \\
\hline$\tau \mathrm{H}_{9} \mathrm{C}_{3} \mathrm{~N}_{18} \mathrm{C}_{2}, \tau \mathrm{H}_{10} \mathrm{C}_{6} \mathrm{C}_{7} \mathrm{~S}_{20}, \tau \mathrm{H}_{11} \mathrm{C}_{7} \mathrm{~S}_{20} \mathrm{C}_{4}(84)$ & 950 & 958 \\
\hline$v \mathrm{~N}_{17} \mathrm{~N}_{16}(16), v \mathrm{~S}_{19} \mathrm{C}_{1}, v \mathrm{~S}_{21} \mathrm{C}_{1}(35), \delta \mathrm{S}_{19} \mathrm{C}_{1} \mathrm{~N}_{17}, \delta \mathrm{S}_{21} \mathrm{C}_{1} \mathrm{~N}_{17}(12), \delta \mathrm{H}_{15} \mathrm{~S}_{21} \mathrm{C}_{1}(12), \delta \mathrm{C}_{1} \mathrm{~N}_{17} \mathrm{~N}_{16}, \delta \mathrm{N}_{16} \mathrm{C}_{2} \mathrm{~N}_{18}(15)$ & 1030 & 1035 \\
\hline$v \mathrm{C}_{5} \mathrm{C}_{4}, v \mathrm{C}_{7} \mathrm{C}_{6}(17), \delta \mathrm{H}_{11} \mathrm{C}_{7} \mathrm{~S}_{2} 0(35), \delta \mathrm{H}_{10} \mathrm{C}_{6} \mathrm{C}_{7}(31)$ & 1067 & 1071 \\
\hline$v \mathrm{~N}_{17} \mathrm{~N}_{16}(44), \delta \mathrm{C}_{1} \mathrm{~N}_{17} \mathrm{~N}_{16}, \delta \mathrm{N}_{16} \mathrm{C}_{2} \mathrm{~N}_{18}(19), \delta \mathrm{N}_{17} \mathrm{~N}_{16} \mathrm{C}_{2}(10)$, & 1090 & 1096 \\
\hline$v \mathrm{~N}_{18} \mathrm{C}_{2}, v \mathrm{C}_{3} \mathrm{C}_{4}, v \mathrm{C}_{6} \mathrm{C}_{5}(30), v \mathrm{~N}_{17} \mathrm{~N}_{16}(14), \delta \mathrm{N}_{17} \mathrm{~N}_{16} \mathrm{C}_{2}(16)$ & 1114 & 1139 \\
\hline$v \mathrm{C}_{3} \mathrm{C}_{4}, v \mathrm{C}_{6} \mathrm{C}_{5}(24), \delta \mathrm{H}_{9} \mathrm{C}_{3} \mathrm{~N}_{18}(15) \delta, \mathrm{C}_{6} \mathrm{C}_{5} \mathrm{C}_{4}(18)$ & 1210 & 1229 \\
\hline$v \mathrm{C}_{5} \mathrm{C}_{4}, v \mathrm{C}_{7} \mathrm{C}_{6}(20), \delta \mathrm{H}_{9} \mathrm{C}_{3} \mathrm{~N}_{18}(52)$ & 1251 & 1314 \\
\hline$v \mathrm{C}_{5} \mathrm{C}_{4}, v \mathrm{C}_{7} \mathrm{C}_{6}(13), \mathrm{H}_{11} \mathrm{C}_{7} \mathrm{~S}_{20}(16), \delta \mathrm{H}_{12} \mathrm{C}_{8} \mathrm{H}_{14}, \delta \mathrm{H}_{13} \mathrm{C}_{8} \mathrm{H}_{12}, \delta \mathrm{H}_{14} \mathrm{C}_{8} \mathrm{H}_{13}(35)$, & 1323 & 1352 \\
\hline$v \mathrm{~N}_{16} \mathrm{C}_{2}, v \mathrm{~N}_{17} \mathrm{C}_{1}, v \mathrm{~N}_{18} \mathrm{C}_{3}(58), v \mathrm{C}_{5} \mathrm{C}_{4}, v \mathrm{C}_{7} \mathrm{C}_{6}(14)$ & 1363 & 1379 \\
\hline$\delta \mathrm{H}_{12} \mathrm{C}_{8} \mathrm{H}_{14}, \delta \mathrm{H}_{13} \mathrm{C}_{8} \mathrm{H}_{12}, \delta \mathrm{H}_{14} \mathrm{C}_{8} \mathrm{H}_{13}(58), \tau \mathrm{H}_{12} \mathrm{C}_{8} \mathrm{C}_{5} \mathrm{C}_{4}, \tau \mathrm{H}_{13} \mathrm{C}_{8} \mathrm{C}_{5} \mathrm{C}_{4}, \tau \mathrm{H}_{14} \mathrm{C}_{8} \mathrm{C}_{5} \mathrm{C}_{4}(17)$ & 1413 & 1428 \\
\hline$v \mathrm{~N}_{16} \mathrm{C}_{2}, v \mathrm{~N}_{17} \mathrm{C}_{1}, v \mathrm{~N}_{18} \mathrm{C}_{3}(10), v \mathrm{C}_{5} \mathrm{C}_{4}, v \mathrm{C}_{7} \mathrm{C}_{6}(44)$ & 1507 & 1509 \\
\hline$v \mathrm{~N}_{16} \mathrm{C}_{2}, v \mathrm{~N}_{17} \mathrm{C}_{1}, v \mathrm{~N}_{18} \mathrm{C}_{3}(59), \delta \mathrm{H}_{9} \mathrm{C}_{3} \mathrm{~N}_{18}(14), \delta \mathrm{H}_{9} \mathrm{C}_{3} \mathrm{~N}_{18}(14)$ & 1581 & 1553 \\
\hline$v \mathrm{~S}_{21} \mathrm{H}_{15}(100)$ & 2586 & 2567 \\
\hline $\mathrm{C}_{8} \mathrm{H}_{1} 2, \mathrm{C}_{8} \mathrm{H}_{13}, \mathrm{C}_{8} \mathrm{H}_{14}(99)$ & 2863 & 2908 \\
\hline$v \mathrm{C}_{8} \mathrm{H}_{1} 2, v \mathrm{C}_{8} \mathrm{H}_{13}, v \mathrm{C}_{8} \mathrm{H}_{14}(99)$ & 2950 & 2957 \\
\hline$v \mathrm{C}_{3} \mathrm{H}_{9}, v \mathrm{C}_{6} \mathrm{H}_{10}, v \mathrm{C}_{7} \mathrm{H}_{11}(95)$ & 3072 & 3069 \\
\hline$v \mathrm{C}_{3} \mathrm{H}_{9}, v \mathrm{C}_{6} \mathrm{H}_{10}, v \mathrm{C}_{7} \mathrm{H}_{11}(99)$ & 3093 & 3108 \\
\hline
\end{tabular}

${ }^{1} \mathrm{H}-\mathrm{NMR}$ spektrumu için, aromatik protonlar (H10 ve H11) deneysel olarak 7.96 ve 7.12 ppm'de, teorik olarak 7.89 ve $7.22 \mathrm{ppm}$ 'de sinyaller verir. Metil grubu protonları (H12, H13, H14), ${ }^{13} \mathrm{C}-\mathrm{NMR}$ spektrumunda olduğu gibi yüksek bölgelerde NMR sinyallerini verir. Çalıșmamızda, metil protonlarından kaynaklanan NMR sinyalleri deneysel olarak 2.50 ppm'de teorik olarak 2.76-2.45 ppm aralıklarında gözlenmiştir. Bileşiğinin deneysel ve teorik ${ }^{1} \mathrm{H}$ ve ${ }^{13} \mathrm{C}$ NMR sonucu Tablo 3 'de verilmiștir. ${ }^{1} \mathrm{H}-$ ve ${ }^{13} \mathrm{C}-\mathrm{NMR}$ kimyasal kayma değerleri beklenen bölgelerde bulunduğu söylenebilir.

Tablo 3. Sentezlenen molekül TMS'ye göre hesaplanmış ve deneysel ${ }^{1} \mathrm{H}$ - ve ${ }^{13} \mathrm{C}-\mathrm{NMR}$ izotropik kimyasal kayma değerleri

\begin{tabular}{|c|c|c|c|}
\hline No & Deneysel & DFT/6-311+G(d,p)/DMSO & Fark/DMSO \\
\hline C1 & 164.39 & 172.25 & -7.86 \\
\hline C2 & 186.56 & 184.49 & 2.07 \\
\hline C3 & 159.69 & 157.19 & 2.50 \\
\hline C4 & 134.32 & 146.67 & -12.35 \\
\hline C5 & 148.45 & 158.67 & -10.22 \\
\hline C6 & 132.21 & 136.22 & -4.01 \\
\hline C7 & 134.79 & 148.08 & -13.29 \\
\hline C8 & 14.16 & 14.83 & -0.67 \\
\hline H9 & 8.74 & 9.77 & -1.03 \\
\hline H10 & 7.12 & 7.22 & -0.10 \\
\hline H11 & 7.96 & 7.89 & 0.07 \\
\hline H12 & 2.50 & 2.45 & 0.05 \\
\hline H13 & 2.50 & 2.50 & 0.00 \\
\hline H14 & 2.50 & 2.76 & -0.26 \\
\hline H15 & 14.01 & 4.89 & 9.12 \\
\hline
\end{tabular}

Sentezlenen yeni bileşiğin $\mathrm{R}$ değeri değerlendirildiğinde; DFT/B3LYP/6311+G(d.p) için; ${ }^{13} \mathrm{C}$ : $0.99,{ }^{1} \mathrm{H}: 0.34$ olduğu görülmüştür. Bulunan standart hata oranı $\delta$ calc=a $\delta$ exp+b formülüne göre hesaplanmıștır (Şekil 4). Teorik ve deneysel karbon ve proton kimyasal kayma oranları arasında $a, b$ ve $R$ değerine göre doğrusal bir korelasyon gözlenmiștir. İncelenen molekül için hesaplanan ve deneysel olarak elde edilen değerler arasında bir uyum görülmüştür. Buna karşın, SH protonu için hesaplanan değerler ile deneysel değerler arasında beklenenden daha yüksek bir fark ortaya çıkmıștır. Bunun nedeni 1,3,4Tiyadiazol halkasındaki $\mathrm{S}-\mathrm{H}$ protonunun asidik özellik taşıması gösterilebilir. Proton ayrıldıktan sonra $S$ atomu üzerinde kalan elektron çifti halka üzerinde delokalize olmuştur. Bu da oluşan anyonun kararlılığını artırır, dolayısıyla SH protonunu asidik yapar.

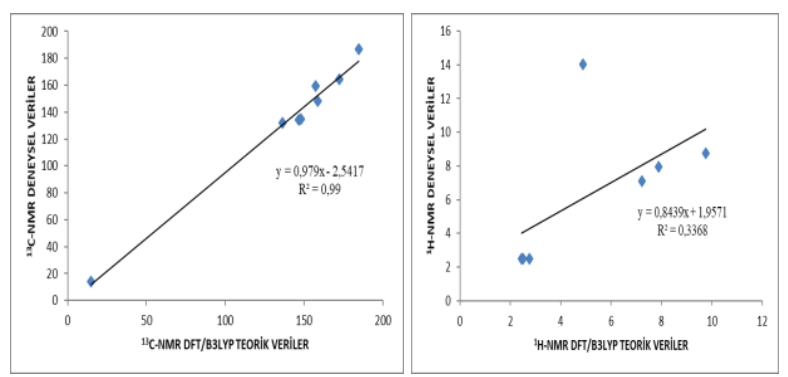

Şekil 4. Molekülün DFT/B3LYP(DMSO) yöntemiyle teorik ve deneysel ${ }^{1} \mathrm{H}$ - ve ${ }^{13} \mathrm{C}$-NMR kimyasal kayma değerlerinin karşılaştırılması

\section{3. Çizgisel olmayan optik özellikler (NLO)}

Polarizebilite ve hiperpolarizebilite, elektrik alanındaki uygulandığı alanda sistemin yanıtını karakterize eder. Elektrik polarizasyonu, atomik ve moleküler sistemlerin temel bir özelliğidir. NLO materyalleri, optik iletişim, optik bilgi işlem, optik anahtarlama ve dinamik görüntü işleme gibi optoelektronik alanındaki gelecekteki potansiyel uygulamaları ile son yıllarda ilgi çekmiștir. Yüksek moleküler hiperpolarizebiliteleri nedeniyle, organik 
materyaller önemli derecede NLO özelliği gösterir. NLO aktivitesi üzerine farklı inorganik, organik ve organometalik moleküler sistemler çalıșılmıștır [41, 42]. Moleküler yapılar ve lineer olmayan optik özellikler arasındaki ilişkileri araştırmak için, toplam statik dipol momenti $(\boldsymbol{\mu})$, bağll polarizebilite $(\boldsymbol{\alpha})$, polarizebilite anizotropisi $(\boldsymbol{\Delta} \boldsymbol{\alpha})$, temel durumdaki hiperpolarizebilite ( $\boldsymbol{\beta})$ değerleri DFT/B3LYP/6311+G(d.p) temel seviyesine göre hesaplanmıştır. Elde edilen değerler Tablo 4'de verilmiştir.

Tablo 4. Molekülün (B3LYP/6-311+G(d,p)) hesaplanan dipol moment, polarizebilite ve hiperpolarizebilite değerleri

\begin{tabular}{|c|c|}
\hline & DFT/B3LYP \\
\hline $\boldsymbol{\mu}_{\mathrm{x}}$ & 3.6407 debye \\
$\boldsymbol{\mu}_{\mathrm{y}}$ & -1.5456 debye \\
$\boldsymbol{\mu}_{\mathrm{z}}$ & 0.8052 debye \\
$\boldsymbol{\mu}_{\text {Toplam }}$ & 4.0363 debye \\
$\boldsymbol{\alpha}$ & $30.91 \times 10^{-24} \mathrm{esu}$ \\
$\boldsymbol{\Delta \alpha}$ & $34.664 \times 10^{-24} \mathrm{esu}$ \\
$\boldsymbol{\beta}$ & $9.945 \times 10^{-24} \mathrm{esu}$ \\
\hline
\end{tabular}

Tablo 4'deki verilere göre, hesaplanan dipol momenti 4.0363 Debye olarak gözlemlenmiştir. B3LYP/6$311+G(d, p)$ temel setine göre eldilen $\alpha, \Delta \alpha$ ve $\beta$ parametreleri sırasıyla $30.91 \times 10^{-24}$ esu, $34.664 \times 10^{-24}$ esu ve $9.945 \times 10^{-24}$ esu olarak hesaplanmıștır. Ürenin $\boldsymbol{\beta}$ değeri $0.77 \times 10^{-30}$ esu olarak bilinmektedir. Elde edilen hiperpolarizabilite değeri üre ile karşılaştırıldığında, sentezlenen molekülün önemli NLO aktivitesine sahip olduğu söylenebilir.

\subsection{Mulliken yüklerinin incelenmesi}

Popülasyon analizi yöntemlerinden en yaygın olarak kullanılanı Mulliken yük dağılımı yöntemidir [43]. Bu yöntem atomik orbitallerin lineer kombinasyonuyla moleküler orbitallerin elde edilmesine dayanır. Fakat bu dağılım her bir elementin elektronegativitesini tam olarak yansıtmaz [44-46]. Bazı aşırı durumlarda bir orbitalde negatif elektron nüfusu verebilir veya bir orbitalde ikiden fazla elektron hesaplayabilir. Sonuç olarak Mulliken yükleri deneysel sonuçları nicel olarak tahmin etmekten ziyade nitel bir takım tahminler yapmak için de kullanılır. Sentezlenen yeni bileşiğin atomik yüklerini hesaplamak için Mulliken yoğunluk analizi DFT/B3LYP/6-311+G(d,p) yöntemiyle hesaplanmıştır (Tablo 5). Bu sonuçlara göre; negatif yükler çalışılan molekülün elektronegatifliği en yüksek olan $O$ ve $C$ atomları üzerinde toplanmıştır.

Tablo 5. Sentezlenen yeni bileşiğin teorik hesaplanan mulliken atomik yük değerleri

\begin{tabular}{|l|c|c|ccc|}
\hline \multicolumn{7}{|c|}{ B3LYP } & B3LYP & & \\
\hline C1 & -0.204 & C8 & -0.913 & H15 & 0.057 \\
C2 & 0.185 & H9 & 0.140 & N16 & -0.246 \\
C3 & 0.718 & H10 & 0.130 & N17 & 0.142 \\
C4 & 0.150 & H11 & 0.167 & N18 & 0.169 \\
C5 & -0.373 & H12 & 0.174 & S19 & 0.126 \\
C6 & 0.258 & H13 & 0.153 & S20 & -0.356 \\
C7 & -0.238 & H14 & 0.148 & S21 & -0.386 \\
\hline
\end{tabular}

\subsection{Moleküler elektrostatik potansiyeli}

Bir molekül etrafındaki olası etkileșim alanlarının yanı sıra hidrojen bağlama etkileşimlerinin belirlenmesi moleküler elektrostatik potansiyeli için çok güçlü bir tanımlayıcıdır [47]. MEP, hedef moleküllerde elektrofilik ve nükleofilik bölgeleri tahmin ederek, incelenen bir sistemin reaktivitesini belirleyebilir. MEP'nin mavi rengi ile temsil edilen pozitif bölge nükleofilik bir bölgedir, kırmızı renkle temsil edilen negatif bölge ise bir elektrofilik bölge ile ilişkilidir (Şekil 5). MEP, en negatif potansiyelin oksijen atomu civarında olduğunu gösterirken, azot ve kükürt az miktarda negatif potansiyel taşır. Diğer tüm protonların küçük pozitif potansiyeli vardır.

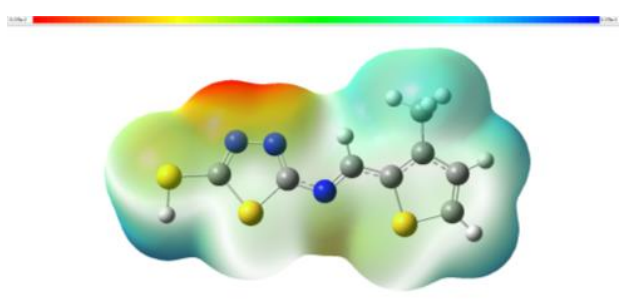

Şekil 5. Molekülün DFT/B3LYP(DMSO) yöntemiyle elde edilmiş moleküler elektrostatik potansiyeli

\section{Tartışma ve Sonuç}

5-Merkapto-2-(3-metil-tiyofen-2-il-metilidenamino)1,3,4-tiyadiazol bileşiği, 2-Amino-5-merkapto-1,3,4tiyadiazol ile 3-metil-2-tiyofen-karboksialdehidin reaksiyonundan sentezlenmiştir. Bu bileșiğin yapısı IR, ${ }^{1} \mathrm{H}$-NMR ve ${ }^{13} \mathrm{C}$-NMR spektroskopik yöntemleri kullanılarak aydınlatılmıștır. Teorik olarak IR, ${ }^{1} \mathrm{H}-$ NMR ve ${ }^{13} \mathrm{C}$-NMR spektral değerleri hesaplanmış ve elde edilen bu değerler deneysel verilerle mukayese edilmiștir. Deneysel ve teorik verilerin uyumlu olduğu gözlemlenmiștir. Teorik olarak molekülün (B3LYP/6-311+G(d,p)) dipol moment, polarizebilite ve hiperpolarizebilite değerleri hesaplanmış ve elde edilen sonuçlar referans madde olarak üre ile karşılaştırılmış hiperpolarizebilite değeri ürenin hiperpolarizebilite daha iyi olduğu görülmüştür. Sentezlenen molekülün önemli çizgisel olmayan optik özelliğe sahiptir. Ayrıca, son olarak, molekülün Mulliken yükleri ve moleküler elektrostatik potansiyeli teorik olarak hesaplanmıştır. Sonuç olarak, sentezlenen yeni molekül için hem deneysel hem de teorik yöntemlerle yapısal, spektroskopik, elektronik ve elektriksel karakterizasyon çalışmaları yapılmıștır.

\section{Kaynakça}

[1] Gür, M., Muglu, H., Çavus, M. S., Güder, A., Sayıner, H. S., Kandemirli, F. 2017. Synthesis, Characterization, Quantum Chemical Calculations and Evaluation of Antioxidant Properties of 1,3,4-Thiadiazole Derivatives İncluding 2- and 3-Methoxy Cinnamic Acids. Journal of Molecular Structure. 1134, 40-50. 
[2] Gür, M., Şener, N., Muglu, H., Çavus, M. S., Ozkan, O. E., Kandemirli, F., Şener, İ. 2017. New 1,3,4Thiadiazole Compounds İncluding Pyrazine Moiety: Synthesis, Structural Properties and Antimicrobial Features, Journal of Molecular Structure. 1139, 111-118.

[3] Yang, S. J., Lee, S. H., Kwak, H. J., Gong Y. D. 2013. Regioselective Synthesis of 2-Amino-Substituted 1,3,4-Oxadiazole and 1,3,4-Thiadiazole Derivatives via Reagent-Based Cyclization of Thiosemicarbazide Intermediate, The Journal of Organic Chemistry. 78 (2), 438-444.

[4] Farghaly, T. A., Abdallah, M. A., Masaret, G. S., Muhammad Z. A. 2015. New and Efficient Approach for Synthesis of Novel Bioactive $[1,3,4]$ Thiadiazoles İncorporated with 1,3Thiazole Moiety, European Journal of Medicinal Chemistry. 97, 320-333.

[5] Gür, M., Şener, N., Kaştas, Ç. A., Ozkan, O. E., Muğlu, H., Elmaswaria, M. A. M. 2017. Synthesis and Characterization of Some New Heteroaromatic Compounds Having Chirality Adjacent to a 1,3,4-Thiadiazole Moiety and Their Antimicrobial ActivitiesJournal of Heterocyclic Chemistry. 54 (6), 3578-3590.

[6] Aliabadi, A., Eghbalian, E., Kiani, A. 2013. Synthesis and Evaluation of The Cytotoxicity of a Series of 1,3,4-Thiadiazole Based Compounds as Anticancer Agents, Iranian Journal of Basic Medical Sciences. 16, 1133-1138.

[7] Mohammadi-Farania, A., Heidarian, N., Aliabadi, A. N. 2014. N-(5-Mercapto-1,3,4-Thiadiazol-2yl)-2-Phenylacetamide Derivatives: Synthesis and in-vitro Cytotoxicity Evaluation as Potential Anticancer Agents, Iranian Journal of Pharmaceutical Research. 13 (2), 487-492.

[8] Kaur, H., Kumar, S., Vishwakarma, P., Sharma, M., Saxena, K. K., Kumar, A. 2010. Synthesis and Antipsychotic and Anticonvulsant Activity of Some New Substituted oxa/thiadiazolylazetidinonyl/thiazolidinonyl carbazoles, European Journal of Medicinal Chemistry. 45, 2777-2783.

[9] Bhatia, R., Sharma, A., Kaundal, A. A. 2014, Review on 1, 3, 4-Thiadiazole Derivatives, Indian Journal of Pharmaceutical Sciences. 4 (3), 165172.

[10] Gupta, J. K., Yadav, R. K., Dudhe, R., Sharma, P. K. 2010. Recent Advancements in the Synthesis and Pharmacological Evaluation of Substituted 1,3,4-Thiadiazole Derivatives, International Journal of PharmTech Research. 2, 1493-1507.

[11] Yar, M. S., Akhter, M. W. 2009. Synthesis and Anticonvulsant Activity of Substituted Oxadiazole and Thiadiazole Derivatives, Acta Poloniae Pharmaceutica. Drug Research. 66 (4), 393-397.
[12] Hafez, H. N., Hegab, M. I., Ahmed-Farag, I. S., ElGazzar, A. B. A. 2008. A Facile Regioselective Synthesis of Novel Spiro-Thioxanthene and Spiro-Xanthene-90,2-[1,3,4] Thiadiazole Derivatives as Potential Analgesic and AntiInflammatory Agents, Bioorganic \& Medicinal Chemistry. 18, 4538-4543.

[13] Poorrajab, F., Ardestani, S. K., Emani, S., Behrouzi-Fardmoghadam, M., Shafiee, A., Foroumadi, A. 2009. Nitroimidazolyl-1,3,4thiadiazole-Based Antileishmanial Agents: Synthesis and in vitro Biological Evaluation, European Journal of Medicinal Chemistry. 44, 1758-1762.

[14] Balaji, K., Bhatt, P., Mallika, D., Jha, A. 2015. Design, Synthesis and Antimicrobial Evaluation of Some Mannich Base Derivative of 2(2Substituted)-5-aminothiadiazoles, International Journal of Pharmacy and Pharmaceutical Sciences. 7 (11), 145-149.

[15] Gomha, S. M., Kheder, N. A., Abdelhamid, A. 0., Mabkhot, Y.N. 2016. One Pot Single Step Synthesis and Biological Evaluation of Some Novel Bis(1,3,4-Thiadiazole) Derivatives as Potential Cytotoxic Agents, Molecules. 21, 1532.

[16] Pattan, S. R., Kekare, P., Dighe, N. S., Nirmal, S. A., Musmade, D. S., Parjane, S. K., Daithankar, A. V. 2009. Synthesis and Biological Evaluation of Some 1,3,4-Thiadiazoles, Journal of Chemical and Pharmaceutical Research. 1 (1), 191-198.

[17] Asif, K. A., Himaja, M., Sunil, M. V., Jagadeesh, K. P., Sikarwar, M. S. 2011. One-Pot Synthesis and Antitubercular Activity of 2-Amino-5-Aryl-5HThiazolo [4,3-B]-1,3,4-Thiadiazoles, International Research Journal of Pharmacy. 2 (1), 153-158.

[18] Balaji, K., Bhatt, P., Mallika, D., Jha, A. 2015. Design, Synthesis and Antimicrobial Evaluation of Some Mannich Base Derivative of 2(2Substituted)-5-aminothiadiazoles, International Journal of Pharmacy and Pharmaceutical Sciences. 7 (11), 145-149.

[19] Zhao, H. C., Shi, Y. P., Liu, Y. M., Li, C. W., Xuan, L. N. Wang, P., Zhang, K., Chen B. Q. 2013. Synthesis and antitumor-evaluation of 1,3-selenazolecontaining 1,3,4-thiadiazole derivatives, Bioorganic \& Medicinal Chemistry Letters. 23, 6577-6579.

[20] Frisch, M. J., Trucks, G. W., Schlegel, H. B., Scuseria, G. E., Robb, M. A., Cheeseman, J. R., Scalmani, G., Barone, V., Mennucci, B., Petersson, G. A., Nakatsuji, H., Caricato, M., Li, X., Hratchian, H. P., Izmaylov, A. F., Bloino, J., Zheng, G., Sonnenberg, J. L., Hada, M., Ehara, M., Toyota, K., Fukuda, R., Hasegawa, J., Ishida, M., Nakajima, T., Honda, Y., Kitao, O., Nakai, H., Vreven, T., Montgomery, J. A., Vreven, T.Jr., Peralta, J.E., 
Ogliaro, F., Bearpark, M., Heyd, J. J., Brothers, E., Kudin, N., Staroverov, V. N., Kobayashi, R., Normand, J., Raghavachari, K., Rendell, A., Burant, J. C., Iyengar, S. S., Tomasi, J., Cossi, M., Rega, N., Millam, J. M., Klene, M., Knox, J. E., Cross, J. B., Bakken, V., Adamo, C., Jaramillo, J., Gomperts, R., Stratmann, R. E., Yazyev, O., Austin, A. J., Cammi, R., Pomelli, C. J., Ochterski, W., Martin, L. R., Morokuma, K., Zakrzewski, V. G., Voth, G. A., Salvador, P., Dannenberg, J. J., Dapprich, S., Daniels, A. D., Farkas, O., Foresman, J. B., Ortiz, J. V., Cioslowski, J., and Fox, D. J., 2009. Gaussian Inc. (Wallingford, CT).

[21] Keith, T., Millam, J. 2009. GaussView, Version 5, R Dennington, Semichem Inc, Shawnee Mission, KS.

[22] Becke, A. D. 1993. Density-functional thermochemistry. III. The role of exact Exchange, The Journal of Chemical Physics. 98, 5648.

[23] Lee, C., Yang, W., Parr, R. G. 1988. Development of the Colle-Salvetti correlation-energy formula into a functional of the electron density, Physical Review B .37, 785.

[24] Jamroz, M. H. 2004. Vibrational Energy Distribution Analysis VEDA4 (Warsaw).

[25] Jamroz, M. H., Dobrowolski, J. C. 2001. Potential energy distribution (PED) analysis of DFT calculated IR spectra of the most stable $\mathrm{Li}, \mathrm{Na}$, and $\mathrm{Cu}(\mathrm{I})$ diformate molecules, Journal of Molecular Structure. 475, 565-566.

[26] London, F. 1937. The quantic theory of interatomic currents in aromatic combinations, Journal de Physique et Le Radium. 8. 397-409.

[27] McWeeny, R. 1962. Perturbation Theory for Fock-Dirac Density Matrix, Physical Review. 126. 1028.

[28] Wolinski, K., Hilton, J. F. Pulay, P. 1990. Efficient Implementation of the Gauge-Independent Atomic Orbital Method for NMR Chemical Shift Calculations, Journal of the American Chemical Society. 112. 8251-8260.

[29] Cheeseman, J. R., Trucks, G. W., Keith, T. A., Frisch, M. J. 1996. A Comparison of Models for Calculating Nuclear Magnetic Resonance Shielding Tensors, The Journal of Chemical Physics. 104. 5497-5509.

[30] Avcı, D., Başoglu, A., Atalay, Y. 2009. Theoretical analysis of vibrational spectra and scaling-factor of 2-aryl-1,3,4-oxadiazole derivatives, International Journal of Quantum Chemistry. $109,328-341$.

[31] Dege, N., Şenyüz, N., Batı, H., Günay, N., Avcl, D., Tamer, Ö., Atalay, Y. 2014. The synthesis, characterization and theoretical study on nicotinic acid [1-(2,3-dihydroxyphenyl) methylidene]hydrazide, Spectrochimica Acta Part A. 120, 323-331.

[32] Tamer, Ö., Avcl, D., Atalay, Y. 2014. Calculations of electronic structure and nonlinear optical parameters of 4-methoxybenzaldehyde-Nmethyl-4-stilbazolium tosylate, Journal of Applied Spectroscopy. 80, 971-982.

[33] Tao, Y., Han, L., Han, Y., Liu, Z. 2015. Experimental and theoretical studies on the vibrational spectra of trans-3-phenylacryloyl chloride, Spectrochimica Acta Part A. 137, 892898.

[34] Koşar, B., Albayrak, C. 2011. Spectroscopic investigations and quantum chemical computational study of (E)-4-methoxy-2-[(ptolylimino)methyl]phenol, Spectrochimica Acta Part A. 78, 160-167.

[35] Pearson, R. G., 1986. Absolute electronegativity and hardness correlated with molecular orbital theory, in: Proceeding of the National Academy of Sciences, 83, 8440-8441.

[36] Jamróz, M. H. 2004. Vibrational energy distribution analysis: VEDA 4 program, Warsaw.

[37] Demir, S., Dincer, M., Cukurovali, A., Yilmaz, I. 2016. Synthesis, characterization, and theoretical studies on N'-furan-2ylmethylene-N[4-(3-methyl-3-phenylcyclobutyl)-thiazol-2-yl]chloro-acetic acid hydrazide, Molecular Crystals and Liquid Crystals. 629 (1), 44-60.

[38] Foresman, J. B., Frisch, E., 1993. Exploring Chemistry with Electronic Structure Methods, Gaussian Inc, Pittsburgh, PA, USA.

[39] Pihlaja, K., Kleinpeter, E. 1994. Carbon-13 NMR Chemical Shifts in Structural and Sterochemical Analysis, VCH Publishers, Deerfield, Beach.

[40] Kalinowski, H. O., Berger, S., Braun, S. 1988. Carbon-13 NMR Spectroscopy, John Wiley \& Sons, Chichester.

[41] Avcl, D., Cömert, H., Atalay, Y. 2008. Ab initio Hartree-Fock calculations on linear and secondorder nonlinear optical properties of new acridinebenzothiazolylamine chromophores, Journal of Molecular Modeling. 14, 161-169.

[42] Turhan Irak Z, Gümüș S, 2017. Heterotricyclic compounds via click reaction: A computational study. Noble International Journal of Scientific Research, 1(7), 80-89.

[43] Mulliken RS, 1955. Electronic population analysis on LCAO-MO molecular wave functions. Journal of Chemical Physics, 23: 1833-1840.

[44] Reed, A. E., Weinstock, R. B., Weinhold, F. 1985. Natural population analysis. The Journal of Chemical Physics, 735. 
[45] Reed, A. E., Weinhold, F. 1985. Natural localized molecular orbitals. The Journal of Chemical Physics, 1736.

[46] Reed, A. E., Curtiss, L. A., Weinhold, F. 1988. Intermolecular interactions from a natural bond orbital, donor-acceptor viewpoint. Chemical Reviews. 899.
[47] Scrocco, E., Tomasi, J. 1979. Electronic molecular structure, reactivity and intermolecular forces: an heuristic interpretation by means of electrostatic molecular potentials, Advances in Quantum Chemistry. 11, 115-193. 